\title{
Diacylglycerol and Triacylglycerol as Responses in a Dual Response Surface-Optimized Process for Diacylglycerol Production by Lipase-Catalyzed Esterification in a Pilot Packed-Bed Enzyme Reactor
}

\begin{abstract}
Diacylglycerol (DAG) and triacylglycerol (TAG) as responses on optimization of DAG production using a dual response approach of response surface methodology were investigated. This approach takes the molecular equilibrium of DAG into account and allows for the optimization of reaction conditions to achieve maximum DAG and minimum TAG yields. The esterification reaction was optimized with four factors using a central composite rotatable design. The following optimized conditions yielded $48 \mathrm{wt} \%$ DAG and $14 \mathrm{wt} \%$ TAG: reaction temperature of $66.29{ }^{\circ} \mathrm{C}$, enzyme dosage of $4 \mathrm{wt} \%$, fatty acid/glycerol molar ratio of 2.14, and reaction time of $4.14 \mathrm{~h}$. Similar results were achieved when the process was scaled up to a $10 \mathrm{~kg}$ production in a pilot packed-bed enzyme reactor. Lipozyme RM IM did not show any significant activity losses or changes in fatty acid selectivity on DAG synthesis during the 10 pilot productions. However, lipozyme RM IM displayed higher selectivity toward the production of oleic acid-enriched DAG. The purity of DAG oil after purification was 92 wt \%.
\end{abstract}

Keyword: Diacylglycerol, dual response, optimization, response surface methodology, packed-bed reactor, lipozyme RM IM, Rhizomucor miehei lipase, esterification, pilot plant 